\title{
Expectativas sobre a sobrevivência ao câncer de pacientes internados em unidades de clínica
}

\author{
Cancer survival expectations of patients admitted to inpatient clinical units
}

Expectativas sobre la supervivencia al cáncer de pacientes ingresados en unidades de hospitalización

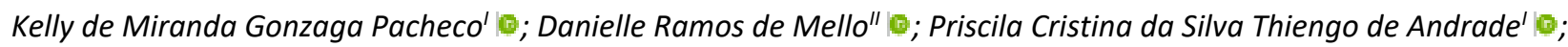

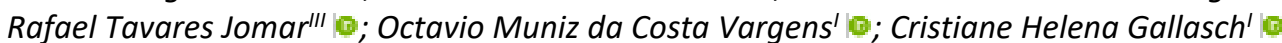 \\ 'Universidade do Estado do Rio de Janeiro, Rio de Janeiro, RJ, Brasil; "Universidade de São Paulo, Ribeirão Preto, SP, Brasil; \\ I'IInstituto Nacional do Câncer, Rio de Janeiro, RJ, Brasil
}

\begin{abstract}
RESUMO
Objetivo: analisar as expectativas de pacientes internados devido ao diagnóstico de câncer sobre o término do tratamento e a sobrevivência à doença. Método: estudo qualitativo e descritivo realizado com 32 pacientes internados em um hospital universitário. A coleta de dados ocorreu entre abril e junho de 2019 por meio de entrevista semiestruturada e a técnica para tratamento dos dados foi a análise de conteúdo. Resultados: observou-se predomínio de expectativas sobre de autonomia para realizar atividades do cotidiano, relacionando à retomada dos papéis sociais e às mudanças de hábitos após o tratamento. Conclusões: é necessário reconhecer as expectativas do sobrevivente ao câncer para auxiliá-lo em direção ao protagonismo acerca de suas decisões sobre sua vida futura e tratamento, incentivando a autonomia na construção do seu processo de saúde.
\end{abstract}

Descritores: Enfermagem; Oncologia; Neoplasias; Sobrevivência; Expectativa de Vida.

\begin{abstract}
Objective: to analyze the expectations of hospital inpatients diagnosed with cancer as to the end of treatment and their surviving the disease. Method: in this qualitative, descriptive study of 32 inpatients at a university hospital, data were collected by semi-structured interview between April and June 2019 and treated by content analysis. Results: expectations for autonomy in performing daily activities predominated, i.e., for resuming social roles and changing habits after treatment. Conclusions: cancer survivors' expectations must be acknowledged in order to assist them towards taking the lead in decisions about their future life and treatment and to encourage autonomy in constructing their health process.

Descriptors: Nursing; Oncology; Neoplasms; Survival; Life Expectancy.
\end{abstract}

\section{RESUMEN}

Objetivo: analizar las expectativas de los pacientes hospitalizados debido al diagnóstico de cáncer en cuanto al término del tratamiento y la supervivencia a la enfermedad. Método: estudio cualitativo y descriptivo realizado junto a 32 pacientes ingresados en un hospital universitario. La recolección de datos se realizó entre abril y junio de 2019 a través de entrevista semiestructurada y la técnica para el tratamiento de los datos fue el análisis de contenido. Resultados: Se observó que predominan expectativas sobre tener autonomía para realizar las actividades diarias, relacionado a la reanudación de los roles sociales y a los cambios de hábitos tras el tratamiento. Conclusiones: Es necesario reconocer las expectativas del superviviente al cáncer para asistirlo en el protagonismo sobre sus decisiones en cuanto a su vida futura y su tratamiento, incentivando la autonomía en la construcción de su proceso de salud.

Descriptores: Enfermería; Oncología; Neoplasias; Supervivencia; Expectativa de Vida.

\section{INTRODUÇÃO}

O câncer é, atualmente, uma das principais causas de mortalidade no mundo, representado por um conjunto de doenças nas quais há crescimento celular desordenado, com tendência infiltrativa dos tecidos adjacentes ${ }^{1,2}$. Devido a essas características, tem estigma de uma enfermidade incurável, com possibilidade inevitável de morte, perda da independência física, autonomia, vitalidade e ainda aumento do risco de ocorrência de dor e mortalidade ${ }^{2}$.

Em 2018, registrou-se mais de 18 milhões de novos casos de câncer e a morte de mais de 9,6 milhões de pessoas devido a doença. No Brasil, o Instituto Nacional de Câncer (INCA) estimou para cada ano do triênio 2020-2022, com exceção do câncer de pele não-melanoma, a incidência de 625 mil casos novos de câncer. Os tipos de cânceres mais incidentes serão os de próstata (65.840) e de mama (66.280) além dos cânceres de cólon e reto, pulmão, estômago, colo do útero, cavidade oral, sistema nervoso central, leucemia e esôfago ${ }^{1}$.

Sendo assim, conhecer quais as expectativas quanto a sobrevivência dos portadores de câncer é essencial para o planejamento de ações de enfermagem que visem ao adequado atendimento de suas necessidades, entendendo que o sobrevivente do câncer vivencia sentimentos de angústia, por não conseguir partilhar os anseios que lhe causam 
desconforto físico e mental. Assim, neste contexto, estabeleceu-se como objetivo analisar as expectativas dos pacientes com diagnóstico de câncer internados em enfermarias clínicas sobre o término do tratamento e a sobrevivência à doença.

\section{REVISÃO DE LITERATURA}

A transição demográfica observada nos últimos anos nos países em desenvolvimento, demandou a estruturação de políticas públicas de prevenção e de promoção de saúde, por causar impactos importantes na saúde da população e está associado com a elevação dos índices de prevalência e de incidência de doenças crônicas não transmissíveis, com destaque para o câncer ${ }^{1}$.

Como visto anteriormente, o câncer tem se tornado uma das doenças responsáveis por uma grande quantidade de óbitos em todo o mundo. Mesmo em países com maior acesso ao serviço de saúde, um dos principais problemas é o diagnóstico tardio, dificultando o tratamento e a sobrevida. A detecção precoce e o tratamento efetivo proporcionam a possibilidade de os indivíduos continuarem trabalhando e apoiando os familiares, reduzindo o impacto da doença na qualidade de vida e uma vez que a efetividade do tratamento é maior nos estágios iniciais ${ }^{3}$.

De acordo com a National Coalition for Cancer Survivorship, o sobrevivente ao câncer é aquela pessoa que recebe o diagnóstico em algum momento de sua vida 4 . Já o The National Cancer Institute define, além da pessoa com câncer, a família e os próprios cuidadores como sobreviventes ${ }^{3}$.

Com essas informações, há de se considerar que, tão difícil quanto sobreviver ao câncer, é vencer os estigmas e estereótipos que a doença carrega, a qual afeta emocionalmente as pessoas que o vivenciam, assim como os seus familiares. Nesse sentido, o diagnóstico de câncer provoca vários sentimentos, inquietações e preocupações nas pessoas, por se tratar de uma doença ameaçadora à vida. Estar com câncer vai além da dor física e do desconforto emocional, sendo necessário a compreensão diferenciada para o cuidado. Prestar assistência a pessoa com câncer exige do profissional de saúde uma preocupação com os aspectos emocionais, sociais e psicológicos para manutenção de sua qualidade de vida ${ }^{5}$.

Os sobreviventes de câncer representam uma nova realidade nos serviços de saúde. Assim, para prestar cuidados adequados a esta crescente população, os profissionais de saúde devem conhecer as suas necessidades, a fim de desenvolver as intervenções adequadas. Neste contexto, a exigência dirigida aos profissionais de saúde é ampliada, sugerindo intervenções direcionadas aos processos educacionais e apoio psicossocial, pessoa e família, visando ao bemestar e qualidade de vida após o tratamento de câncer.

A literatura descreve a necessidade, desde a fase de implementação do protocolo terapêutico, a garantia de uma efetiva comunicação entre equipe multidisciplinar em saúde, paciente e familiares/cuidadores, a fim de facilitar a compreensão da situação de saúde, promover autonomia durante o tratamento e permitir a construção da perspectiva de futuro ${ }^{4}$.

\section{MÉTODO}

Pesquisa descritiva, com abordagem qualitativa, que pretendeu captar informações dos pacientes com diagnóstico de câncer e retratar a essência das expectativas após o término do tratamento, em uma perspectiva que incluísse sentimentos, valores e percepções destes sobre suas atividades e as repercussões na sua qualidade de vida ${ }^{6}$.

O estudo foi desenvolvido em três unidades de internação clínica geral de um hospital universitário do município do Rio de Janeiro. No período da coleta de dados, entre abril e junho de 2019, cerca de 648 pessoas estiveram internadas nas unidades pesquisadas, com motivos de internação diversos, predominando distúrbios cardiovasculares, gastrointestinais, hematológicos e oncológicos. A escolha de unidades clínicas não oncológicas foi justamente pela presença desses pacientes "fora de clínica", que nos leva a refletir sobre a necessidade do preparo formal dos profissionais que Ihe prestam assistência. Foram incluídos na amostra indivíduos com diagnóstico de câncer e ciente do quadro clínico, nível de consciência e orientação preservados. Foram excluídos pacientes com rebaixamento de nível de consciência ou alteração da orientação, de acordo com a avaliação diária registrada em prontuário pela equipe de enfermagem, que demostraram não conhecer o próprio diagnóstico e apresentando dificuldade na compreensão do estudo.

Mediante o aceite do participante e da assinatura do Termo de Consentimento Livre e Esclarecido em duas vias, realizou-se a coleta dos dados à beira do leito, a partir de uma entrevista semiestruturada, com o auxílio de um instrumento de caracterização sociodemográfica e clínica (idade, sexo escolaridade, profissão/ocupação, renda, afastamento de atividades laborais, rede de apoio e diagnóstico) e contendo a seguinte questão norteadora: Quais atividades, compromissos ou relações que espera retomar ao término do tratamento? As respostas foram anotadas em formato de lista em impresso próprio pela pesquisadora principal.

A análise dos dados sociodemográficos e clínicos foi realizada por estatística descritiva simples, com apoio dos softwares Microsoft Office Excel ${ }^{\oplus}$ e Statistical Package for the Social Sciences ${ }^{\oplus}$ (SPSS v.20.0). As informações relatadas sobre as atividades e expectativas foram listadas e submetidas à analise obedecendo a metodologia da análise de 
conteúdo: Pré-análise: com organização do material e composição do corpus do estudo; Exploração do material: no qual os dados são transformados sistematicamente e agregados em unidades de registro (UR) que possuíam características em comum e posteriormente, agrupadas e classificadas em unidades de significação (US) ou temas; Tratamento dos resultados: com inferência e interpretação, buscando embasar as análises e dar sentido à interpretação ${ }^{7}$. Assim, após a leitura das respostas, foi possível delimitar 103 US que foram agrupadas em três categorias: Desempenho dos papéis sociais, Autonomia para realizar atividades do cotidiano diário e Mudanças de hábitos para melhorar a saúde e a convivência.

O protocolo de pesquisa foi aprovado pelo Comitê de Ética em Pesquisa sob CAAE no 06240919.0.0000.5282, obedecendo às Resoluções vigentes do Conselho Nacional de Saúde.

\section{RESULTADOS E DISCUSSÃo}

Participaram do estudo 32 pacientes com idade entre 26 e 82 anos. Observou-se predomínio de homens (56,3\%), com ensino fundamental (56,3\%). A maioria afirmou estar afastado de suas atividades laborais (78,1\%), sendo que $12,6 \%$ expõem não ter renda, no momento da realização do estudo. Destaca-se predomínio do diagnóstico de leucemia (25\%) e linfoma (18,8\%). Todos relataram ter apoio da família sendo que, $21,89 \%$ reconhecem os amigos e $9,38 \%$ os colegas de trabalho como redes de apoio.

A superioridade de casos do sexo masculino corrobora com os dados encontrados pelo Instituto Nacional de Câncer (INCA $)^{1}$. Destaca-se, com relação a esse dado, a necessidade de ter especial atenção a esse grupo, uma vez que muitos homens sentem vergonha de procurar ajuda, por influência de questões culturais, mitos ou tabus, retardando o diagnóstico de doenças ${ }^{8}$. O grau de escolaridade dos entrevistados predominante foi o ensino fundamental, confirmando o perfil de pacientes atendidos em outro serviço público de quimioterapia, e atuando como potencial fator de atraso na identificação precoce do câncer, por dificultar o acesso e a assimilação de informações sobre prevenção, diagnóstico, controle e tratamento ${ }^{9}$.

Devido ao acometimento por doenças do sistema osteomuscular e tecido conjuntivo, a dor foi apresentada como motivo principal da internação de muitos deles. A dor é uma das queixas mais frequentes em pacientes com diagnóstico de câncer e responsável pelo seu sofrimento e de sua família. Outros estudos mostraram que pacientes com câncer foram internados em enfermarias gerais e a dor foi o sintoma mais comum ${ }^{10}$.

Destaca-se o alto índice de afastamento das atividades do cotidiano pela maioria dos entrevistados, mesmo sendo esperado devido à internação hospitalar. Alguns participantes referiram não ter possibilidade de voltar ao trabalho ou terem perdido o emprego por conta do câncer, tratamento ou efeitos colaterais. Além disso, afirmaram ter dificuldades para encontrar um emprego devido ao cronograma de tratamento e seus efeitos.

Sobreviventes de câncer relatam prejuízo profissional por conta da doença devido a incapacidade de prosseguir com suas carreiras, por adiar planos de graduação ou pela impossibilidade de viajar a trabalho ${ }^{11}$. Neste sentido, ressaltase a importância de discutir a situação econômica e social da família nas reuniões de equipe, para se consideram melhores possibilidades de suporte e encaminhamento específico a serviços de apoio.

Os participantes também foram questionados quanto às suas expectativas de atividades, compromissos ou relações que esperavam retomar após o término do tratamento, por meio de perguntas abertas. Houve uma grande variação de respostas listadas, analisadas e categorizadas como: Desempenho dos papéis sociais, Autonomia para realizar atividades do cotidiano diário e Mudanças de hábitos para melhorar a saúde e a convivência. As respostas são demonstradas na Figura 1 e as categorias discutidas a seguir.

\section{Desempenho dos papéis sociais}

Com relação ao desempenho dos papeis sociais, a maioria dos participantes manifestou a vontade de estar e/ou conviver com a família, além de poder criar filhos netos ou outros dependentes.

A literatura mostra que a família possui grande efeito na melhora e na qualidade de vida dos pacientes em tratamento, sendo muitas vezes, a principal fonte de apoio social, emocional e afetivo, um suporte essencial no processo saúde-doença ${ }^{11}$. Um estudo recente avaliou a qualidade de vida de pacientes com câncer em diferentes períodos de tratamento e demonstrou que a qualidade de vida está diretamente relacionada ao suporte social oferecido ${ }^{12}$.

Destaca-se que prejuízos na capacidade funcional decorrentes da doença ou de seu tratamento podem afetar a capacidade de desempenho nas atividades de vida diária e prática, o que inclui as relações sociais ${ }^{13}$.

A família possui um grande efeito na melhoraria e a qualidade de vida dos pacientes que realiza tratamento para qualquer tipo de câncer. Seu suporte e presença é essencial, devido à sua importância que não se restringe apenas ao apoio emocional e afetivo, mas também no auxílio aos cuidados à saúde ${ }^{14}$. 


\begin{tabular}{|llll|}
\hline Respostas & N & $\mathbf{f ( \% )}$ & Classificação \\
\hline Estar /conviver com a família & 8 & 25,0 & Desempenho dos papéis sociais \\
Criar filhos/netos/dependentes & 5 & 15,62 \\
Estudar & 2 & 6,30 \\
Casar & 1 & 3,16 \\
Dirigir & 1 & 3,16 \\
Ser feliz & 1 & 3,16 \\
\hline Trabalhar & 19 & 59,38 Autonomia para realizar atividades \\
Praticar atividades físicas/lazer & 14 & 43,75 do cotidiano diário \\
Frequentar igreja/práticas religiosas & 13 & 40,63 \\
Cuidar da casa & 5 & 15,62 \\
Conviver com amigos & 4 & 12,50 \\
Cuidado à própria saúde & 2 & 6,30 \\
Cuidar de animais de estimação & 1 & 3,16 \\
Independência & 1 & 3,16 \\
Pagar contas & 1 & 3,16 \\
Prova de vida no banco & 1 & 3,16 \\
Conseguir aposentar & 1 & 3,16 \\
\hline Viajar & 4 & 12,50 Mudanças de hábitos para \\
Parar de fumar & 3 & 9,36 \\
Fazer uma horta/plantas & 3 & 9,36 \\
Entender as pessoas & 1 & 3,16 \\
Ter paz & 1 & 3,16 \\
Falar não para as pessoas & 1 & 3,16 \\
Parar de beber & 1 & 3,16 \\
Mudar do Brasil & 1 & 3,16 \\
Participar de Organização Não-Governamental (ONG) a convivência & 1 & 3,16 \\
\hline
\end{tabular}

FIGURA 1: Atividades, compromissos ou relações que os pacientes esperavam retomar após o término do tratamento. Rio de Janeiro, RJ, Brasil, 2019.

Além disso, os pacientes com câncer apresentam preocupações sobre os seus papeis de pais, tanto para realizar atividades práticas usuais para seus filhos, como também se quanto às preocupações com as consequências emocionais para as crianças ${ }^{15}$. Estudo anterior observou que a maioria dos pacientes (60\%) considerou ter sido extremamente capaz de realizar suas responsabilidades parentais antes do diagnóstico, caindo para $11 \%$ posteriormente ${ }^{16}$.

\section{A autonomia para realizar atividades do cotidiano}

O diagnóstico de câncer, uma doença com tantos estigmas, que carreia sofrimentos e preocupações para quem por ele é acometido quanto para todos que o cercam, uma situação desestruturante, que gera, muitas vezes, mudanças de comportamento, levando-o a se aproximar ou se afastar daqueles que o cercam. É possível supor que o diagnóstico por si só levará ao sujeito a desencadeamento de reflexões e sentimentos, podendo ser influenciados por experiências próprias e de outros, que resultarão em comportamentos de adaptação, cuja finalidade é o enfrentamento do estresse e da ansiedade provocados por esse momento ${ }^{17,18}$.

Nesse sentido, a expectativa pela qualidade de vida e a volta ao trabalho apontam a esperança pelo retorno de uma vida normal e como forma de enfrentar a doença e a internação. A vivência do câncer para os participantes do estudo provocou momentos de incerteza e frustração, no entanto, a partir das falas, pode-se perceber a esperança de acreditar na cura da doença e a volta a suas atividades. Contudo, foi frequente o medo em relação a recidiva e as metástases colocando os indivíduos em alerta para o que pode ser enfrentado no futuro, levando-os a adaptar-se e a sobreviver a essa doença.

Em relação ao trabalho, elemento mais citado dessa categoria, destaca-se que a questão financeira é algo que preocupa os sobreviventes do câncer devido ao impacto na economia da família. Neste estudo, a maioria dos participantes relataram afastamento de suas atividades laborais, presumindo que provavelmente muitos precisaram abdicar do emprego devido a doença. A situação social e a incapacidade da manutenção do emprego são questões que podem gerar algum tipo de agravo aos pacientes com câncer e desafios para seus familiares ${ }^{19}$. Deste modo, é importante que durante o atendimento, a equipe de saúde esteja atenta não somente aos sintomas, mas que os aspectos socioeconômicos sejam abordados para ampliar a atenção a saúde do indivíduo. 
O desejo de praticar atividades físicas e realizar práticas religiosas também destacam-se entre as respostas que emergiram como expectativas de retomada da autonomia. Sabe-se que a prática de exercícios físicos regulares, de intensidade moderada, está relacionada a melhora da capacidade funcional bem como possui efeitos anti-inflamatórios e imunoestimuladores, contribuindo para o melhor prognóstico do paciente com câncer ${ }^{19}$.

A espiritualidade e a religiosidade são recursos largamente utilizados pelos pacientes com câncer, pois o bemestar religioso e espiritual, na maioria dos casos, proporciona um enfrentamento positivo, mudando a maneira de lidar com o diagnóstico e a adesão ao tratamento oncológico ${ }^{20}$. O pensamento positivo relacionado à doença esteve presente entre os participantes, por meio da citação da fé em Deus e na busca pela cura, comportamento já observado também em outros estudos ${ }^{21}$.

\section{Mudanças de hábitos para melhorar a saúde e a convivência}

As mudanças de hábitos para melhorar a saúde e a convivência foram destacados especialmente quando ao desejo de viajar (5 US) e mudanças de hábitos, como parar de fumar (5 US).

A mudança de hábitos vistos como prejudiciais à saúde, emoções negativas e alimentação pouco saudável, e oncogênicos como o fumo, podem expressar um comportamento direcionado pela pressão social anticâncer e mecanismos de defesa psicológicos. Essa alteração de conduta em suas vidas diárias é considerada adequada no processo de superação do problema de saúde. No entanto, também pode ser considerada como parte do processo de negação da doença ${ }^{22}$.

A assistência ao paciente com diagnóstico de câncer, por ser um processo permeado por dúvidas, medo e insegurança, exige atenção especial e cuidado integral de equipe multiprofissional bem treinada e com conhecimento específico dessa população, a fim de atender suas demandas biopsicossociais e espirituais. É necessário um papel proativo dos profissionais de enfermagem na avaliação das necessidades básicas destes pacientes ${ }^{22}$.

Diante dos resultados, percebe-se que o sujeito se torna ativo quando participa das decisões sobre sua própria vida e, pensando no âmbito da autonomia em saúde, quando torna-se elemento central na construção do seu processo de saúde, diferente da lógica que denomina uma pessoa pela sua patologia, ofuscando sua personalidade e sua capacidade de decisão, ou participação - "um paciente". A reflexão sobre a legitimidade e o respeito à autonomia do paciente/usuário, faz surgir o questionamento sobre a valorização da vontade do paciente em busca de uma assistência humanizada. Muitos profissionais encaram o paciente com diagnóstico de câncer como um indivíduo fragilizado, com declínio rápido do estado geral e com dificuldades para aceitar o diagnóstico, principalmente se esse paciente for idoso ${ }^{12}$.

Nesse sentido, destaca-se a necessidade de que, desde o período de formação profissional, o futuro enfermeiro desenvolva um olhar para o indivíduo como ser biopsicossocioespiritual, buscando compreender suas dimensões e proporcionar bem-estar. A busca pela autonomia pelo paciente faz parte do atendimento humanizado que respeita e promove $o$ atendimento das necessidades deste.

\section{Limitações do estudo}

Reconhece-se que uma das limitações do estudo está relacionada ao baixo número de participantes, devido às particularidades das unidades de clínica, não específicas para pacientes oncológicos. Além disso, o estudo foi desenvolvido em um único serviço de saúde, limitando generalizações a pacientes hospitalizados em instituições semelhantes ao cenário deste estudo. Acredita-se que seria apropriado realizar outros estudos, comparando a outros serviços, principalmente especializados em câncer com pacientes hospitalizados e em regime de tratamento e/ou acompanhamento ambulatorial.

\section{CONCLUSÃO}

Os resultados refletem a percepção dos sobreviventes do câncer sobre suas expectativas, bem como a esperança em relação a sua autonomia após o término do tratamento. O relato dos pacientes indicou a necessidade promoção da qualidade de vida e o retorno de suas atividades, ainda que indicando a subjetividade dessas escolhas, vinculadas a valores pessoais e influenciado pelas repercussões do processo saúde-doença.

Além disso, a retomada dos papéis sociais, dando visibilidade a questões familiares e aos impactos financeiros relacionados a doença, e a mudança dos hábitos para melhoria da saúde, demonstram a necessidade de retorno a vida e ao alívio do sofrimento causado a partir da privação das atividades pela doença.

Nesse contexto, faz-se necessário conhecer e reconhecer essas expectativas dos sobreviventes ao câncer em relação ao tratamento e a vida futura, para uma assistência de enfermagem individualizada e de maior qualidade. Assim, considera-se importante o apoio profissional aos pacientes e seus familiares, ignorando pré-julgamentos e suposições e se permitindo ouvir, a partir da escuta atenta e acolhedora de forma a promover o cuidado humanizado e proporcionando bem-estar. 


\section{REFERÊNCIAS}

1. Ministério da Saúde (Br). Secretaria de Atenção à Saúde, Instituto Nacional de Câncer José Alencar Gomes da Silva. Estimativa 2020: Incidência do Câncer no Brasil. Rio de Janeiro: INCA; 2019 [cited 2020 Jul 16]. Available from:

https://www.inca.gov.br/publicacoes/livros/estimativa-2020-incidencia-de-cancer-no-brasil.

2. Sales CA, Almeida CSL, Wakiuchi J, Piolli KC, Reticena K. I Survived câncer: phenomenological analysis of the survivors' language. Texto Contexto Enferm [Internet], 2014 [cited 2020 Jul 16]; 23(4):880-8. DOI: http://dx.doi.org/10.1590/010407072014002050013.

3. McGuire S. World Cancer Report 2014. Advances in Nutrition [Internet], 2016 [cited 2019 Sep 30]; 7(2):418-19. DOI: http://dx.doi.org/10.3945/an.116.012211.

4. Delevatti VF, Salaza VM, Schneider TMD, Castro EK. Life expectancy and future prospects in cancer patients: systematic review. Psicol. Pesq [Internet], 2018 [cited 14 jul 20]; 12 (1): 15-22. DOI: http://dx.doi.org/10.24879/2018001200100328.

5. Lima SF, Silva RGM, Silva VSC, Pasklan ANP, Reis LMCB, Silva UC. Social representations about cancer among relatives of patient undergoing oncological treatment. REME Rev. Min. Enferm. [Internet], 2016 [cited 2020 Jul 14]; 20:e967. Available from: http://pesquisa.bvsalud.org/portal/resource/pt/biblio-835275.

6. Polit DF, Beck CT. Fundamentos da pesquisa em enfermagem: avaliação de evidências para a prática de enfermagem. $7 \underline{a}$ ed. Porto Alegre (RS): ArtMed; 2011.

7. Bardin L. Análise de conteúdo. São Paulo: Edições 70; 2011.

8. Vieira RC, Moraes MTM, Sarmento LMC, Ferreira ADC, Muñoz RLS. Demand for palliative care in general clinic wars. Rev. Cien. e Est. Acad. de Medicina [Internet], 2017 [cited 2020 Oct 29]; (8):20-40. Available from: https://periodicos.unemat.br/index.php/revistamedicina/article/view/1888/2230.

9. Ferreira JIC, Martins ERC, Ramos RCA, Costa CMA, Alves RN, Lima B. Comprehensive men's health care policy: challenges for nursing. Rev. enferm. UERJ [Internet], 2016 [cited 2020 Jul 15]; 24(6):e7631. DOI: http://dx.doi.org/10.12957/reuerj.2016.7631.

10. Figueiredo JF, Souza VJ, Coelho HV, Souza RS. Quality of life on oncological patients under palliative care. RECOM Rev. enferm. Centro-Oeste Min. [Internet], 2018 [cited 2019 Sep 14]; 8:e2638. DOI: http://doi.org/10.19175/recom.v8i0.2638.

11. Banegas MP, Schneider JL, Firemark AJ, Dickerson JF, Kent EE, de Moor JS, et al. The social and economic toll of cancer survivorship: a complex web of financial sacrifice. J. Cancer Surviv [Internet], 2019 [Cited 2019 Sep 15]; 13:406-17. DOI: https://doi.org/10.1007/s11764-019-00761-1.

12. Yang L, Song WP, Chen ZL, Wang Y, Chen YY, Hua YH, et al. Correlation between social support and quality of life in patients with breast cancer at different periods of treatment. Zhonghua Zhong Li Za Zhi [Internet], 2017 [cited 2020 Jul 16]; 39(3):202-6. DOI: http://dx.doi.org/10.3760/cma.j.issn.0253-3766.2017.03.009.

13. Germano CMR, Bonato D, Maion VH, Avó LRS de, Melo DG, Fontanella BJB. Possíveis novos determinantes da qualidade de vida de pacientes com câncer de tireoide tratado: um estudo qualitativo. Cien. Saude Colet. [Internet], 2016 [cited 16 jul 20]; 21(8):2451-62. DOI: http://doi.org/10.1590/1413-81232015218.18142015.

14. Freire MEM, Costa SFG, Lima RAG, Sawada NO. Health-related quality of life of patients with cancer in palliative care. Texto contexto - enferm. [Internet]. 2018 [cited 16 jul 20]; 27(2):e5420016. DOI: http://dx.doi.org/10.1590/0104070720180005420016.

15. Dutra TL, Guimarães VS, Silva CH, Neves YF, Simião DS, Silva PAV. A importância da assistência familiar a pacientes com câncer: um relato de experiência. REINPEC Rev. Interd. Pens. Científico [Internet], 2018 [cited 15 Jul 20]; 4(20):165-80. Available from: http://www.reinpec.org/reinpec/index.php/reinpec/article/view/195/157.

16. Park EM, Deal AM, Check DK, Handson LC, Reeder-Hayes KE, Mayer DK, et al. Parenting concerns, quality of life, and psychological distress in patients with advanced cancer. Psycho-Oncology [Internet]. 2015 [cited 2019 Sep 16]. DOI: https://doi.org/10.1002/pon.3935.

17. Moore CW, Rauch PK, Baer L, Piri WF, Murel AC. Parenting changes in adults with cancer. Cancer [Internet]. 2015 [cited 2019 Aug 10]; 121(19):3551-6. DOI: https://doi.org/10.1002/cncr.29525.

18. Souza MMS de, Arruda AJCG de, Rodrigues FA, Silva GM da, Santos FS, Vasconcelos DIB de. Sentimentos de idosos hospitalizados pelo câncer: expectativas sobre alta hospitalar e a influência familiar. Rev. enferm. UFPE [Internet], 2016 [cited $15 \mathrm{Jul}$ 20]; 10 (10): 3720-26. Available from: https://pesquisa.bvsalud.org/portal/resource/pt/bde-30100.

19. Meneguin S, Matos TDS, Ferreira MLSM. Perception of cancer patients in palliative care about quality of life. Rev. Bras. Enferm. [Internet], 2018 [cited 2020 Jul 15]; 71(4):1998-2004. DOI: http://dx.doi.org/10.1590/0034-7167-2017-0360.

20. Gurgel DC, Junior VLMC, Nogueira IC, Neto PP. Physical activity and cancer: nutritional interventions for better prognosis. Motri [Internet]. 2018 [cited 2019 Sep 30]; 14(1):398-404. Available from: http://www.scielo.mec.pt/scielo.php?script=sci_arttext\&pid=S1646-107X2018000100062\&lng=pt.

21. Ouro GC, Sodré BC, Figueiredo EGC, Souto LAD, Fernandes MTT, Fernandes MT. Análise da influência da fé, espiritualidade e religião no prognóstico de pacientes com câncer. Cien. Saude Colet. [Internet], 2018 [cited 28 Sep 19]; 7(2):502. Available from: https://rsc.revistas.ufcg.edu.br/index.php/rsc/article/view/101.

22. Ribeiro GS, Campos CS, Anjos ACY dos. Spirituality and religion as resources for breast cancer copíng. Rev. Cuidado é Fundamental [Internet], 2019 [cited 2020 Jul 16]; 11(4):849-56. DOI: http://dx.doi.org/10.9789/2175-5361.2019.v11i4.849-856.

23. Fruet IMA, Dalmolin GL, Bresolin JZ, Andolhe R, Barlem ELD. Moral Distress Assessment in the Nursing Team of a HematologyOncology Sector. Rev. Bras. Enferm [Internet], 2019 [cited 2020 Jul 16]; 72(Suppl 1):58-65. DOI:

http://dx.doi.org/10.1590/0034-7167-2017-0408. 